\title{
Giant fluctuations in diffusion processes
}

\author{
Doriano Brogioli, Alberto Vailati and Marzio Giglio \\ Istituto Nazionale per la Fisica della Materia and Dipartimento di Fisica, Università di Milano, \\ via Celoria 16, Milano, Italy
}

Received 21 October 1999

\begin{abstract}
Concentration fluctuations in a homogeneous mixture are in general small and confined to the molecular lengthscale. It has been recently predicted that stressed fluids should exhibit anomalously large fluctuations. We will show here that anomalously large fluctuations also occur when macroscopic concentration gradients relax to the uniform state via diffusion. The measurements have been taken by low angle static light scattering and shadowgraphs. We find that at larger wavevectors the amplitude of the fluctuations diverges as $q^{-4}$. It is also found that gravity stabilizes at a constant value the fluctuations below a critical wavevector. We will present data on a mixture close to a consolution critical point. Recent results will also be presented on ordinary liquid mixtures, a polymer and a protein solution. These new results have been obtained by means of a quantitative shadowgraph technique. They confirm that giant fluctuations are always associated with mass flow due to diffusion across a macroscopic gradient.
\end{abstract}

\section{Introduction}

Fluctuations of thermodynamic quantities in an equilibrium system occur as an inevitable consequence of its finite temperature. For ordinary fluid mixtures concentration fluctuations occur at molecular lengthscales, and their mean square amplitude is minimal. Indeed their presence is best evidenced by the scattering of light, a process that is ideal to detect fluctuations. The scattered intensity is hardly dependent on scattering angle, thus proving that the relevant scale is at a molecular level. Thermodynamic conditions are seldom met in real conditions, and macroscopic mass flows are induced by the presence of concentration gradients (the ordinary Fick diffusion flow), or by an applied temperature gradient (the Soret effect) or pressure gradient (barodiffusion). A question naturally arises as whether concentration fluctuations in all these non-equilibrium cases should remain the same as in the equilibrium thermodynamic case.

In this contribution we will show that non-equilibrium fluctuations under time dependent free diffusion processes become enormous, both in amplitude and in their spatial extent. They become readily observable with the naked eye with the aid of a phase rendering technique like shadowgraphs. Quantitative measurements have also been conducted with static low angle light scattering. At variance with equilibrium mixtures, the scattered intensity falls very rapidly at larger scattering angles. This proves that longer wavelength fluctuations have larger amplitudes, and that the spatial extent of the fluctuations is much larger than in equilibrium. We will show however that gravity plays a striking role, locking the amplitude of the fluctuations to a constant value for wavelengths larger than a critical one.

We will first describe earlier results obtained with a binary liquid mixture close to a consolution critical curve (Vailati and Giglio 1997). This peculiar choice was made in order to have an almost ideal free diffusion system, with two miscible phases separated by 
a flat sharp boundary at the beginning of the experiment. The measurements have been performed by means of a qualitative shadowgraph technique and by quantitative low angle static light scattering. We will then present more recent data obtained by means of quantitative shadowgraph analysis on free diffusion processes in mixtures of ordinary liquids, a polymer solution and a protein solution (Brogioli et al 1999). These newer data give strong evidence that the giant, long wavelength fluctuations are indeed a general feature associated with mass flow due to diffusion across a macroscopic concentration gradient.

\section{The earlier measurements on a quasi-critical mixture}

In planning the first experiment (the one on a critical system), we had a number of preoccupations in mind. First, we had to select a system that could best approximate the ideal free diffusion scheme, that is a system that at time $t=0$ is made up by two miscible liquid phases, separated by a very sharp, flat meniscus. The requirement that the concentration change across the transition layer should be as sharp as possible was dictated by the fact that the amplitude of the non-equilibrium fluctuations depends strongly on the magnitude of the concentration gradient. We also wanted a system where the macroscopic concentration gradient would depend on one variable only, with no accidental stirring of the transition layer and unwanted accidental perturbations. Experimentally it is fairly hard to prepare a fluid system according to the above prescription. We would like to remind the reader that free diffusion (that is the study of the time evolution of the concentration profiles during the remixing) has been a well established technique for the determination of the diffusion coefficient $D$, and a number of methods have been developed to prepare the initial boundary (Tyrrell 1961). Some of them are rather laborious, and none of them works really well. We then decided to use a trick to prepare a system with a sharp boundary. We selected a binary liquid mixture close to a consolution curve. Below the critical temperature the system phase separates into two immiscible phases, with a sharp flat meniscus between them. The system is then rapidly brought above the critical temperature, and now the two phases become miscible, and diffusion across the initially sharp meniscus begins. The next problem was the choice of the direction of observation. In the classical free diffusion measurements, the direction of observation was horizontal, so that the time evolution of the gradient could be followed. In the present experiment we wanted to look at the fluctuations on top of the larger macroscopic disturbances caused by the gradient itself. So we decided to maintain the main optical beam axis vertical both for the shadowgraph and the scattering technique. In this way there is no contribution to the signals due to the presence of the macroscopic gradients, and both techniques are sensitive to the fluctuations only.

The first qualitative observations were performed by means of the shadowgraph technique. Conceptually the technique is quite simple. A parallel beam of light is sent onto the sample, and the intensity distribution of the outcoming beam at a given distance from the sample is captured by a CCD camera. The phase modulations introduced by a distorting transparent medium are mapped into intensity variations according to the local values of the second order index of refraction derivatives in the directions orthogonal to the optical axis. Stated more simply, the disturbances act as lens-like perturbations, and the shorter their focal length, the stronger the intensity variations onto the receiving screen.

We show in figure 1 a sequence of shadowgraph images obtained during the (inverse) quench from $T_{c}-T=3 \mathrm{~K}$ to $T-T_{c}=1 \mathrm{~K}$ in an aniline-cyclohexane mixture prepared at the critical concentration. We report in figure 1(a) the shadowgraph image obtained before the temperature jump above the critical temperature, and in 1(b) and (c) images taken 100 seconds and 90 minutes after the quench, respectively. Finally in figure 1(d), taken three days after the process was started, the shadow image does not contain any sign of the fluctuations. Notice 


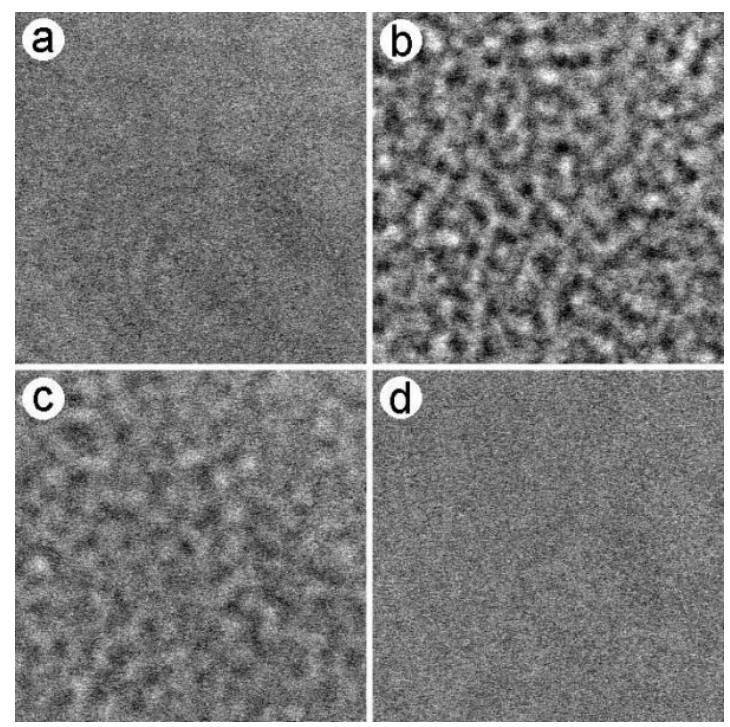

Figure 1. Shadowgraph images of the aniline-cyclohexane binary mixture: (a) was obtained before the start of the diffusion process; (b), (c) and (d) correspond to $100 \mathrm{~s}, 90$ minutes and three days after the start, respectively. The side of each panel is $1 \mathrm{~mm}$ in real space.

that the size of the shadows is microscopic (the side of each panel in figure 1 is $1 \mathrm{~mm}$ wide). While a quantitative analysis of the shadow images is rather complex (and this problem will be discussed later on in the section referring to the newer measurements), it is immediately appreciated from figure 1 that the fluctuations attain their larger value at the very beginning of the diffusion process and they diminish in their intensity as time goes on and the gradient becomes more diffuse. In order to obtain quantitative data we then decided to use low angle light scattering. Scattering is ideal to probe index of refraction fluctuations. It is commonly used to probe fluctuations on smaller lengthscales. Here, since fluctuations grow to rather larger spatial extents, we had to utilize a very low angle static light scattering technique, covering scattering angles from $2^{\prime}$ to $3^{\circ}$. The instrument has been briefly described elsewhere (Carpineti et al 1990). Again the sample is a horizontal liquid slab, $4.5 \mathrm{~mm}$ thick and confined between two thick sapphire plates. The temperature control is performed by controlling the temperature of the sapphire plates by means of Peltier pumps controlled by an electronic servo. The main beam is vertical.

The routine during a data collection sequence is the same used for the shadow images. We first bring the mixture to $T_{c}-T=3 \mathrm{~K}$, and let the phase separation occur. A meniscus is formed, and we let the sample stabilize for a few hours. Then the temperature is set at $T-T_{c}=1 \mathrm{~K}$, and the time constant for the temperature jump is of the order of $100 \mathrm{~s}$. After this time the sample has come to equilibrium at the new temperature, and we take this moment as $t=0$. Scattering measurements are then taken as a function of time (each measurement consists of 31 values of scattered light intensity at 31 scattering angles logarithmically spaced over the instrumental range). The data are shown in figure 2 . The strongest scattering occurs at the beginning of the diffusion process, as in the case of the shadow images. The scattered intensity is presented as a function of the scattering wavevector $q=4 \pi n / \lambda \sin (\theta / 2)$, where $\theta$ is the scattering angle and $\lambda$ the light wavelength. The plots are on a logarithmic scale, and all the curves decay approximately as $q^{-4}$ at larger wavevectors. At smaller wavevectors $q$ the curves saturate to a constant value, and the transition to the fast 


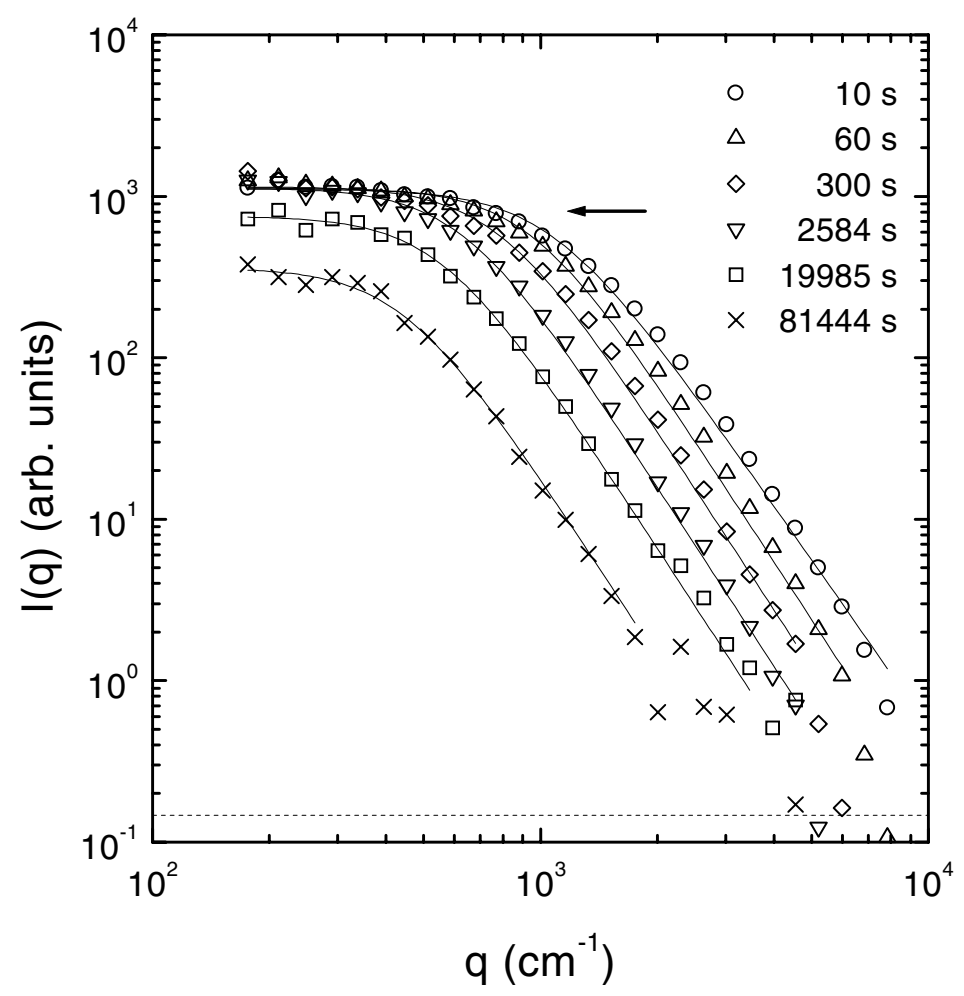

Figure 2. Nonequilibrium scattered intensity distributions against wavevector $q$ at different times during the diffusion process in the aniline-cyclohexane binary mixture. The dashed line represents the intensity scattered by the sample at the end of the process. The arrow marks the predicted level of $I(q=0)$ as calculated from equation (3).

decay occurs at a critical roll-off wavevector that slightly decreases as time goes on. Finally, one can notice that the asymptotic values at smaller wavevectors $q$ coincide at the beginning of the diffusion process, while later on (approximately after one hour) this value begins to fall.

We will analyse these results on the basis of the theoretical predictions. First, the rapid $q^{-4}$ decay is in agreement with the predictions of Law and Nieuwoudt (1989). Indeed the $q^{-4}$ decay can be taken as the signature of the spectrum of non-equilibrium fluctuations (Kirkpatrick et al 1982). The existence of a critical roll-off wavevector is associated with the presence of a gravitational field. It has been shown (Vailati and Giglio 1998) that this wavevector identifies the slowest relaxing mode. Naively speaking, the nonequilibrium concentration fluctuations are generated by velocity fluctuations parallel to the macroscopic concentration gradient. The velocity fluctuations displace parcels of fluid into layers with different concentration, thus giving rise to concentration fluctuations. Two distinct mechanisms are effective in relaxing the fluctuations. At larger wavevectors the controlling mechanism is diffusion, which is associated with time constants $\tau=1 / D q^{2}$. At shorter $q$ gravity is the controlling decay mechanism. Fluctuations in concentration (and therefore in the density) will generate buoyancy forces that will drive the fluctuations in the vertical direction until the associated parcel of fluid is brought into regions of matching density, and the fluctuation then disappears. This buoyancy driven effect becomes faster as the dimension of the fluctuation becomes larger, and therefore becomes 
the dominating mechanism at smaller wavevectors. The roll-off wavevector is given by

$$
q_{R O}=\left(\frac{\beta g \nabla c}{\nu D}\right)^{1 / 4}
$$

where $\beta=\rho^{-1}(\partial \rho / \partial c), v$ is the kinematic viscosity, $D$ the diffusion coefficient and $\nabla c$ the concentration gradient at the midheight of the sample. Notice that in figure 2 the roll-off gradually shifts to smaller values due to the decrease of the concentration gradient as time goes on.

The intensity scattered per unit thickness by a layer with a concentration gradient $\nabla c$ is given by

$$
I(q) \propto\left(\frac{\partial n}{\partial c}\right)^{2} \frac{\nabla c}{\beta g} \frac{1}{1+\left(q / q_{R O}\right)^{4}} .
$$

Since the beam is directed vertically and therefore goes across layers with different values of $\nabla c$, the overall scattered intensity from the sample is the sum of the various contributions from the different layers. We can however approximate the actual concentration profile during the early phases as a layer of constant gradient and limited height. As time goes on the gradient decreases but the width increases as its inverse (the concentration drop across the diffusing layer is constant). Due to the $(\nabla c)^{2}$ dependence, the intensity at larger wavevectors must therefore drop, as shown in figure 2.

Notice also that in the limit $q$ going to 0 the formula reduces to the form

$$
I(q=0) \propto\left(\frac{\partial n}{\partial c}\right)^{2} \frac{\Delta c}{\beta g}
$$

where $\Delta c$ is the concentration difference between the top and bottom layer. Equation (3) is remarkable as it does not depend on any relevant fluid coefficient. Notice that in the early phases, when the diffusing layer is thin compared with the cell thickness, $\Delta c$ is constant. This explains why at first the scattering curves at $q=0$ coincide. They eventually start to drop when diffusion enters the regime of restricted diffusion, and the concentration difference at the boundaries begins to drop. The actual numerical value can be easily determined on the basis of reference values. Since the scattering instrument is calibrated against a standard, we are in the position of showing in figure 2 the calculated theoretical value for $I(q=0)$. This value is reported in figure 2 with an arrow, and one can notice the remarkable agreement with the experimental data.

\section{More recent data on ordinary mixtures and solutions}

The choice of a critical binary mixture did attract some criticism, because it was conjectured that the phenomena reported above could be the result of artifacts associated with phase transitions. It was therefore decided to repeat the experiment on some ordinary mixtures and macromolecular solutions where no contributions from phase transitions or critical scattering could be suspected. We had then to face the problems associated with the preparation of the initial state of the free diffusing system. It became immediately clear that it was not possible to generate systems with transition layers as sharp as those obtainable with the quenching trick used with the quasi-critical system. As a consequence, it was expected that the scattering signals would become weaker, at least at larger wavevectors (because of the $(\nabla c)^{2}$ dependence in equation (2)). We had then to chose a different optical technique, like the shadowgraph, and apply it in the $q$ range around $q_{R O}$, where according to equation (2) the signal is expected to depend on $\nabla c$ rather than on $(\nabla c)^{2}$. 
Luckily the shadowgraph techniques had been recently revisited by D Cannell and coworkers who showed how the method could give fully quantitative results (Wu et al 1995, de Bruyn et al 1996).

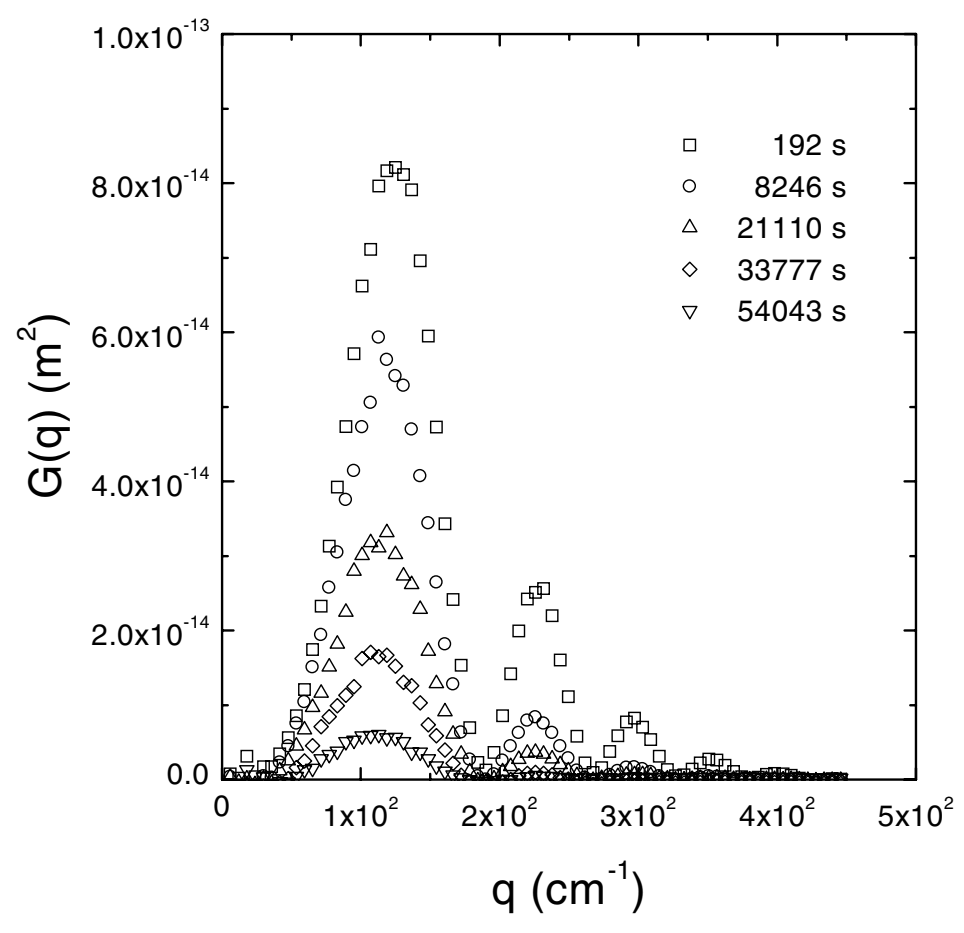

Figure 3. Shadowgraph images power spectrum $G(q)$ against wavevector $q$ measured along the diffusive process in the water-urea sample. The oscillating contribution of the shadowgraph transfer function $T(q)$ is apparent. The oscillations are modulated by the structure factor $S(q)$.

The sample preparation technique of the four systems investigated (all of them are water mixtures or solutions) was to inject the denser solution first into the cell. Then water was carefully layered on top. By so doing the transition layer is smeared out and initially it is somewhat irregular. It was found however that gravity really helps here, and the layers rapidly stack one on top of the other. Incidentally this can be taken as a manifestation of the fact that the slowest diffusing mode is at $q=q_{R} O$, and the longer wavelength irregularities are rapidly ironed out because the buoyancy effects dominate. As long as the diffuse layer is thin compared with the cell height, equation (3) tells that the mean square amplitude of the fluctuations in the plateau region $q \sim 0$ does not change with time. The shadow technique can be arranged to cover this low $q$ region without having the serious stray light problems of low angle scattering.

Raw shadow images are very unimpressive, since what one sees is basically stray contributions due to diffraction effects from dust and other imperfections. The true signal must then be recovered by subtracting the stray contributions, and usually the signal itself is only a few per cent of the raw image signal. If we denote by $I(x, y)$ the image intensity with the fluctuations on and $I_{0}(x, y)$ the blank image (that is the intensity distribution with no fluctuations, but the same cell and optics), one defines the CCD intensity signal as $f(x, y)=\left[I(x, y)-I_{0}(x, y)\right] / I_{0}(x, y)$. We then take the power spectrum of this intensity distribution $G(x, y)=|\mathrm{FFT}[f(x, y)]|^{2}$. 


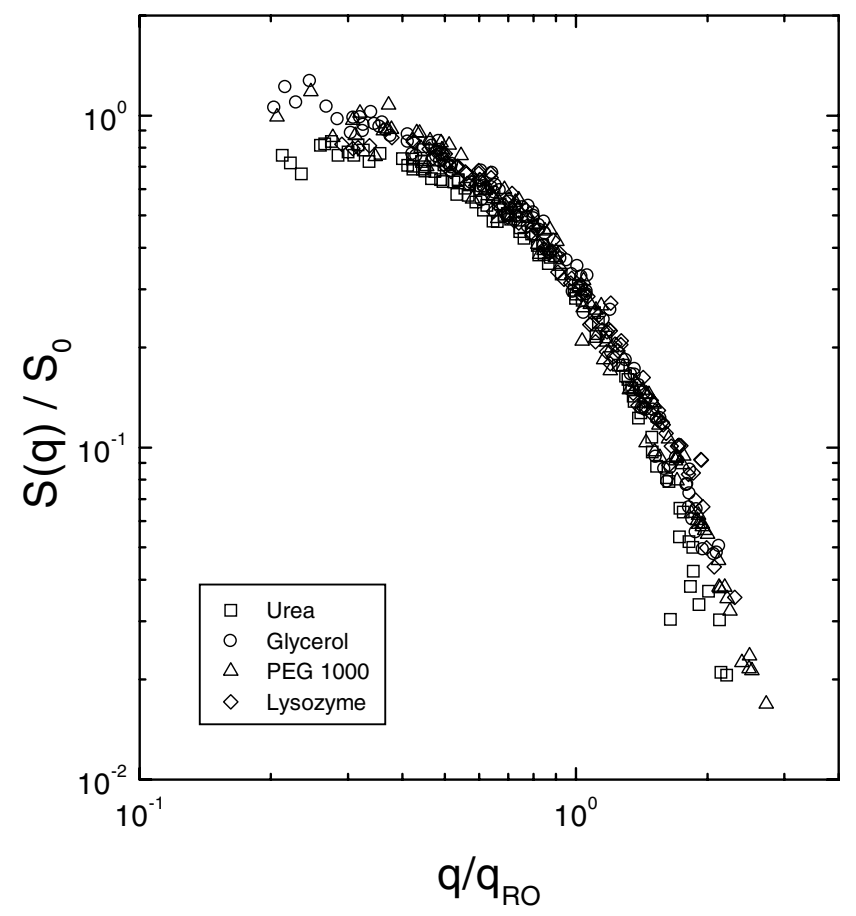

Figure 4. Plot of the scaled static structure factor measured during the free diffusion process in aqueous solutions of urea, glycerol, polyethylene glycol 1000 and lysozyme. The time sequences from individual samples are plotted by scaling the wavevector $q$ with the time-dependent roll-off wavevector $q_{R O}$ calculated from equation (1). The structure factors are scaled by the sampledependent amplitude $I(q=0)$ calculated from equation (3).

It can be shown that the fluctuations structure factor $S(q)$ (the same as measured with light scattering) is given by

$$
G(q)=S(q) T(q)
$$

where $T(q)$ is the shadowgraph transfer function.

Cannell and coworkers (de Bruyn et al 1996) have calculated the instrumental transfer $T(q)$ that accounts both for the geometrical optics effects (the lenslike perturbations) and the wave optics effects, and light source finite size effects. It is found that

$$
T(q)=T_{1}(q) T_{2}(q)
$$

where $T_{1}(q)$ is given by

$$
T_{1}(q)=\left[2 k \sin \left(\frac{q^{2} z}{2 k}\right)\right]^{2}
$$

where $k=2 \pi / \lambda$ is the light wavevector and $z$ is the distance between the fluid slab and the plane of the image captured by the CCD. Notice that the parabolic dependence at smaller $q$ is the geometrical optics limit of the transfer function (the one that accounts for the effects of the lenslike perturbations). $T_{2}(q)$ describes the finite size light source.

We report in figure 3 some of the image power spectra $G(q)$ taken at various times during the diffusion of a water-urea solution. The consequences of the zeros of $T(q)$ are immediately appreciable. In order to obtain the data for $S(q)$ one then has to divide $G(q)$ by $T(q)$. While 
$T_{1}(q)$ can be easily calculated, it is more difficult to account theoretically for the $T_{2}(q)$ term. In order to avoid this problem, we have actually determined experimentally the whole transfer function $T(q)$ by measuring the image power spectrum with a delta correlated calibration sample (basically a screen of small random dots). The derived structure factors can thus be calculated, and typical examples are shown in figure 4 (in this reduction procedure we discard the data that are around the zeros of the transfer function). Indeed figure 4 summarizes a great number of structure factors determined at various instants during the diffusion process and for all the four samples. All the curves have been plotted as a function of the reduced wavevector variable $q / q_{R O}$ that accounts for the evolution of the concentration profiles. During a run, $q_{R O}$ changes as a function of time because of the changes in $\nabla c$ (see equation (1)). The theoretical values for $q_{R O}$ have been calculated from equation (1) by assuming a diffusive evolution of the concentration gradient: $\nabla c \propto t^{-0.5}$. Moreover the measured structure factors have been normalized by their amplitude at $q=0$ calculated from equation (3). The very good collapse of the data onto a master curve does show that individual sets of measurements relative to one mixture do evolve in time as expected, but it also shows that the amplitude of the fluctuations in different systems scales according to equation (3). The nice crowding of the data does indeed provide strong evidence that giant non-equilibrium fluctuations are indeed a genuine manifestation associated with diffusion controlled mass flow across a macroscopic concentration gradient.

In conclusion, we have shown that giant fluctuations are present during diffusion processes in liquid systems including ordinary and near-critical binary mixtures and macromolecular solutions. Two questions naturally arise: in which way do the fluctuations affect the macroscopic mass transfer? In view of the fact that under microgravity conditions the effect is going to be larger, is this going to introduce new and unexpected effects on diffusion-controlled mass transfer processes (for example crystal growth)?

\section{Acknowledgments}

We would like to acknowledge with thanks illuminating discussions with D Cannell on the shadow technique and with P Cicuta on surface scattering from interfaces.

\section{References}

Brogioli D, Vailati A and Giglio M 1999 Phys. Rev. E, submitted

Carpineti M, Ferri F, Giglio M, Paganini E and Perini U 1990 Phys. Rev. A 427347

de Bruyn J R, Bodenschatz E, Morris S W, Trainoff S P, Hu Y, Cannell D S and Ahlers G 1996 Rev. Sci. Instrum. 67 2043

Kirkpatrick T R, Cohen E G D and Dorfman J R 1982 Phys. Rev. A 26995

Law B M and Nieuwoudt J C 1989 Phys. Rev. A 403880

Tyrrell H J V 1961 Diffusion and Heat Flow in Liquids (London: Butterworths)

Vailati A and Giglio M 1997 Nature 390262

-1998 Phys. Rev. E 584361

Wu M M, Ahlers G and Cannell D S 1995 Phys. Rev. Lett. 751743 